\title{
New C-substituted (1S,4S)-2,5-diazabicyclo[2.2.1]heptane derivatives. Preparation utilizing the directed metalation strategy. Application in enantioselective catalysis
}

\author{
Ulrich Jordis,* Martin Kesselgruber, and Sven Nerdinger \\ Institut für Organische Chemie, Technische Universität Wien, Getreidemarkt 9/154, A-1200 \\ Wien, Austria \\ E-mail: Ulrich.Jordis@tuwien.ac.at; Martin.Kesselgruber@post.rwth-aachen.de; \\ Sven.Nerdinger@morphochem.de
}

(received 18 Dec 00; accepted 29 Oct 01; published on the web 06 Nov 01)

\begin{abstract}
New C-substituted derivatives of (1S,4S)-2-methyl-2,5-diazabicyclo[2.2.1]heptane were synthesized utilizing the directed metalation strategy. The absolute configuration of the 3substituted derivative rests on the comparison of the NMR spectra with a product of proven configurational assignment by X-ray crystallographic analysis.
\end{abstract}

Keywords: Enantioselective catalysis, directed lithiation, borolidine reduction

\section{Introduction}

Various N-substituted derivatives of $(1 S, 4 S)$-2,5-diazabicyclo[2.2.1]heptane are known in the literature. ${ }^{1}$ C-Substituted derivatives are rather scarce and include methyl derivatives, ${ }^{2}$ lactams (3-oxo-substituted), ${ }^{3}$ and a 7-hydroxy-7-phenyl-substituted species. ${ }^{4}$ Compounds containing the $(1 S, 4 S)$-2,5-diazabicyclo[2.2.1] heptane moiety have proven to be useful building blocks in organic synthesis, and particularly, in medicinal chemistry. ${ }^{1 \mathrm{~d}, \mathrm{l}, \mathrm{l}, \mathrm{h}, \mathrm{li}, 1 \mathrm{j}, 11}$ Contrary to the conformationally flexible piperazine, the 2,5-diazabicyclo[2.2.1]heptane scaffold is rigid and chiral. The parent compound is synthesized from the naturally occurring amino acid trans-4hydroxy-L-proline. 5 Beak and Hoppe pioneered the directed lithiation of N-protected amines, ${ }^{6}$ a procedure, which allows asymmetric induction in this step when the generally applicable secbutyllithium/(-)-sparteine system is employed for deprotonation. ${ }^{7,8}$

In a recent communication we published the synthesis of novel C-substituted derivatives of $(1 S, 4 S)$-2,5-diazabicyclo[2.2.1] heptane (1) (Scheme 1). This has been achieved by lithiation of the Boc-protected derivative 2 followed by the reaction with suitable electrophiles. ${ }^{9}$ After deprotection the resulting -functionalized amines have been tested as ligands catalyzing the asymmetric addition of diethylzinc to benzaldehyde. ${ }^{10}$ 
In this paper we provide full experimental details of the synthesis and characterization of the products. Furthermore, three of the products were tested as chiral auxiliaries in the borolidineinduced reduction of propiophenone. ${ }^{11}$

\section{Results and Discussion}

The synthesis of 1 started from commercially available trans-4-hydroxy-L-proline, which was Ntosylated and subsequently reduced to $(2 S, 4 S)$-4-hydroxyprolinol. Upon tosylation of both hydroxy groups the reaction with methylamine under pressure formed the additional ring of $\mathbf{1}$. Finally, the $\mathrm{N}$-tosyl group was removed by cleavage with hydrobromic acid.

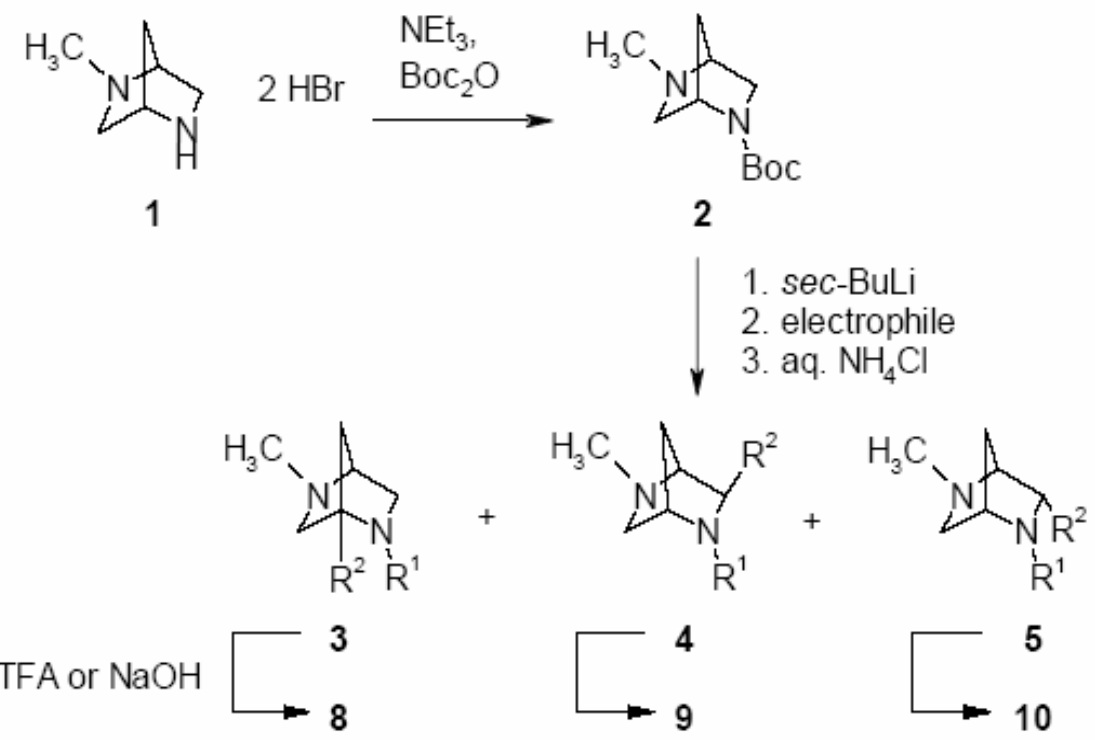

$$
\text { 3, 4, } 5 \mathrm{R}^{1}=\mathrm{Boc} ; \mathbf{8}, \mathbf{9}, 10 \mathrm{R}^{1}=\mathrm{H} \text {; }
$$

\section{Scheme 1}

$$
\mathrm{R}^{2} \text { see Table } 1 \text {. }
$$

In the course of this work this procedure was slightly altered by replacing the two O-tosyl leaving groups with O-mesyl thus slightly improving the yield of the cyclization reaction (92\% vs $86 \%$ in the original procedure). The cyclization was carried out in toluene solution instead of methanol. N-Detosylation was performed in 33\% hydrobromic acid $30 \% \mathrm{HBr} / \mathrm{AcOH}$ in the literature) improving the yield of $1 \cdot 2 \mathrm{HBr}$.

After N-Boc protection of $\mathbf{1}$ with Boc-anhydride/triethylamine the deprotonation of $\mathbf{2}$ was performed with 1.5 equivalents of sec-butyllithium/ $N, N, N^{\prime}, N^{\prime}$-tetramethyl-ethylenediamine (TMEDA) followed by the addition of a suitable electrophilic reactant and furnished the Csubstituted products 3, 4, and/or 5 (Scheme 1). The reactivity of some electrophiles with monocyclic aromatic groups (benzophenone, diphenyldisulfide) appears to depend on the choice of solvent; thus dry diethyl ether instead of tetrahydrofuran (THF) enhanced the regioselectivity 
but dramatically decreased the conversion rate and yield (as evidenced from TLC monitoring of the reactions). For most electrophiles, substitution at position 1 of $\mathbf{2}$ affording $\mathbf{3}$ is favored over substitution at position 3 furnishing 4 and/or 5. Most 3-substituents $\mathrm{R}_{2}$ have been found to adopt the axial orientation as in products 4 (vide infra). As an exception, the group $\mathrm{R}_{2}=1$,1-bis[(3trifluoromethyl)phenyl]-1-hydroxymethyl has been introduced both as axial and equatorial substituent in $\mathbf{4 b}$ and $\mathbf{5 b}$, respectively (Table 1). The lithiated species derived from $\mathbf{2}$ seem to exist in an equilibrium of 1- and 3-deprotonated species before the reaction with the electrophilic reactant furnished either of the three C-substituted products $\mathbf{3}, \mathbf{4}$, and/or $\mathbf{5}$.

Table 1. Yields of 3, 4, 5 obtained from the reaction of $\mathbf{2}$ with electrophiles

\begin{tabular}{ccccc}
\hline Entry & $\mathrm{R}^{2}$ & Yield 3[\%] & Yield 4[\%] & Yield 5 [\%] \\
\hline a & 27 & - & 16 \\
$\mathbf{b}$ & 12 & 12 & 4 \\
$\mathbf{c}$ & 26 & - & 14 \\
$\mathbf{d}$ & 33 & - & 34 \\
$\mathbf{e}$ & 25 & - & 3 \\
$\mathbf{f}$ & - & - & 13 \\
$\mathbf{g}$ & $(4)_{\mathrm{a}}$ & - & - \\
\hline
\end{tabular}

${ }^{\mathrm{a}}$ See text.

The coupling patterns of three versus two methylene groups permitted the distinction between 1- and 3-substituted products 3 and $\mathbf{4}$ and/or 5, respectively. X-Ray diffraction determined the stereochemistry of compound $\mathbf{4} \mathbf{b}^{9}$ and established the equatorial orientation of the 3- substituent. Conventional NMR techniques were not applicable because the coupling constants required for the configurational assignment were within the linewidths of the NMR signals. Comparison of the ${ }^{1} \mathrm{H}$ NMR spectrum of $\mathbf{4} \mathbf{b}$ with those of other 3 -substituted products allowed the unequivocal configurational assignment of these compounds 5a-f. The electrophiles with polycyclic armatic groups (c, e) resulted in predominant substitution at the bridgehead (Table 1), in contrast to the electrophiles with monocycloc aromatic groups (a, d) resulting in low regioselectivity. Surprisingly, with 3,3'-bis(trifluormethyl)benzophenone as electrophile the resulting substituent assumes the equatorial position at $\mathrm{C}-3$.

In the case of $9 H$-thioxanth-9-one as electrophile two different products were obtained depending on the amount of sec-BuLi utilized for deprotonation. With 1.2 equivalents of secBuLi the expected 1-substituted product 3g (Table 1) was isolated in only 4\% yield. Using 1.5 equivalents of sec-BuLi resulted in the formation of the spiro compound 6 ( $24 \%$ yield). This is explained by the intramolecular nucleophilic attack of the O-lithiated intermediate (i.e. the lithium salt of $\mathbf{3 g}$ ) on the carbamate functionality, a mechanism not uncommon in the literature. ${ }^{12}$ The structure 6 was further proven by the formation of 7 upon $\mathrm{LiAlH}_{4}(\mathrm{LAH})$ reduction (Scheme 2). 


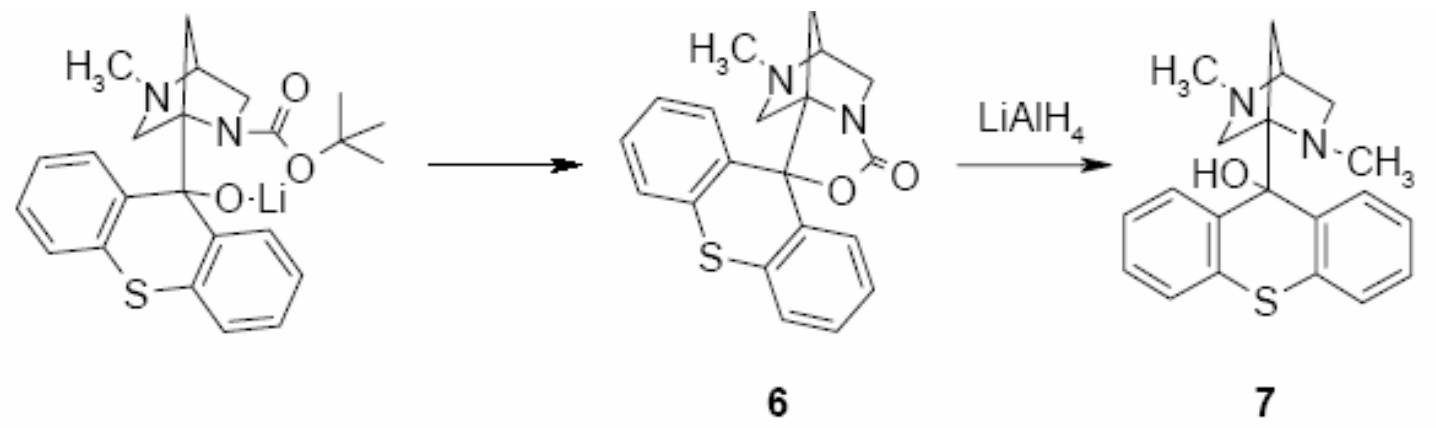

Scheme 2

Table 2. Yields of the deprotection reactions

\begin{tabular}{lll}
\hline Product & Reagent & Yield [\%] \\
\hline 8a & $\mathrm{NaOH}$ & 46 \\
$\mathbf{1 0 a}$ & $\mathrm{NaOH}$ & 47 \\
$\mathbf{8 b}$ & $\mathrm{TFA}$ & 81 \\
$\mathbf{9 b}$ & $\mathrm{NaOH}$ & 71 \\
$\mathbf{8 c}$ & $\mathrm{TFA}$ & 82 \\
$\mathbf{8 d}$ & LAH & 18 \\
$\mathbf{8 e}$ & $\mathrm{TFA}$ & 20 \\
\hline
\end{tabular}

Generally, cleavage of the Boc-group in 3, 4, and 5 was achieved either by treatment with trifluoro acetic acid (TFA), aqueous sodium hydroxide or LAH (Table 2). In some cases the yields were disappointingly low. Boc-cleavage of 3d (featuring a thioether functionality) could not be achieved with sodium hydroxide. Utilization of TFA resulted in partial cleavage of the thioether group and decreased the yield of $\mathbf{8 d}$ to almost zero. A viable solution was the utilization of LAH although the yield of $\mathbf{8 d}$ remained very low.

Application of $(1 S, 4 S)$-2-methyl-2,5-diazabicyclo[2.2.1]heptane derivatives in asymmetric catalysis

Three selected compounds 8a-c were also tested as chiral auxiliaries in the asymmetric reduction of propiophenone by a chiral borolidine species formed in situ prior to addition of the ketone. Borolidine reduced propiophenone to 1-phenyl-1-propanol (Scheme 4), and the resulting enantiomers were analyzed by chiral HPLC. Only with 8a very modest enantiomeric excess was achieved. (Table 3).<smiles>CCC(=O)c1ccccc1</smiles>

Scheme 3 
Table 3. Borolidine reductions

\begin{tabular}{lll}
\hline Ligand $L^{*}$ & ee & Configuration \\
\hline $\mathbf{8 a}$ & 27 & $S$ \\
$\mathbf{8 b}$ & rac. & - \\
$\mathbf{8 c}$ & rac. & - \\
\hline
\end{tabular}

In conclusion, we describe a new class of -amino alcohols prepared by directed metalation of Boc-protected $(1 S, 4 S)$-2,5-diazabicyclo[2.2.1]heptane and subsequent addition to aromatic ketones followed by deprotection.

\section{Experimental Section}

General Procedures. All employed reagents were commercial compounds. (S,trans)-4hydroxyproline was purchased from SIGMA. Dry THF, diethyl ether, and toluene were freshly distilled from sodium/benzophenone ketyl radical. sec-BuLi was purchased from Fluka Corp. and was titrated before use against 2-butanol (in dry toluene, indicated by phenanthroline hydrochloride). Thin-layer chromatography (TLC) was performed using Merck silica gel (60 F254) plates $(0.25 \mathrm{~mm})$. For the monitoring of compounds not detectable under UV light a ninhydrin spray reagent was used. Column chromatography was performed using J. T. Baker silica gel $60(40-63 \mathrm{~m})$. Melting points $(\mathrm{mp})$ were determined using a Kofler hot stage microscope. Proton $\left({ }^{1} \mathrm{H}\right)$ and carbon $\left({ }^{13} \mathrm{C}\right)$ magnetic resonance spectra (NMR) were recorded on a Bruker $200 \mathrm{FS}\left(200 \mathrm{MHz}\right.$ for ${ }^{1} \mathrm{H}, 50 \mathrm{MHz}$ for ${ }^{13} \mathrm{C}$ ) Fourier transform spectrometer, and chemical shifts were expressed in parts per million () relative to tetramethylsilane using solvent signals as an internal reference: DMSO- $d_{6}, 2.50\left({ }^{1} \mathrm{H}\right)$ and $39.5\left({ }^{13} \mathrm{C}\right)$ or $\mathrm{CHCl}_{3}, 7.24\left({ }^{1} \mathrm{H}\right)$ and $77.0\left({ }^{13} \mathrm{C}\right)$; multiplicities: s (singlet), d (doublet), t (triplet), q (quartet), m (multiplet). Reaction temperatures were measured using a digital thermometer inside the reaction vessel. Enantiomeric excess (ee) was determined by chiral HPLC using a Chiralcel OD-H column (4.6 mm x $250 \mathrm{~mm}$, Daicel Chem. Ind. Ltd), detection at $254 \mathrm{~nm}$, pump and detector from Waters. Optical rotations were determined with a Perkin-Elmer polarimeter.

(1S,4S)-2-Methyl-2,5-diazabicyclo[2.2.1]heptane dihydrobromide (1). (2S,4S)-O,O-Dimesyl1-tosyl-4-hydroxy-2-hydroxymethylpyrrolidine (200.0 g, $0.468 \mathrm{~mol})$ were suspended in toluene $(2000 \mathrm{~mL})$ in a $5 \mathrm{~L}$ steel autoclave precooled to $-20^{\circ} \mathrm{C}$. Liquid methylamine was prepared by treating solid sodium hydroxide $(50.0 \mathrm{~g})$ with an aqueous methylamine solution $(40 \%, 300 \mathrm{~mL})$ and condensing the developing methylamine gas in a cool trap at $-20^{\circ} \mathrm{C}$. Liquid methylamine $(58.0 \mathrm{~g}, 1.872 \mathrm{~mol})$ was added, the autoclave was closed and heated to $100^{\circ} \mathrm{C}$ internal temperature. The reaction was finished after 16 hours the pressure decreasing to almost zero indicating the end of the conversion. The reaction mixture (clear solution with some precipitate) was filtered and extracted twice with aqueous sodium hydroxide $(10 \%, 500 \mathrm{~mL}$ each). The 
aqueous phases were extracted with toluene $(2 \times 200 \mathrm{~mL})$, the combined organic phases were washed with saturated brine $(200 \mathrm{~mL})$, dried over sodium sulfate and evaporated affording a yellow oil which was either crystallized by digesting with petroleum ether yielding (1S,4S)-2methyl-5-tosyl-2,5-diazabicyclo[2.2.1] heptane $(114.0 \mathrm{~g}, 91.5 \%)$ or used directly for the detosylation by heating to reflux in hydrobromic acid $(33 \%, 300 \mathrm{~mL})$ for $2 \mathrm{~h}$ followed by evaporation to dryness. The crude product was triturated using diethyl ether/methanol $(2: 1, \mathrm{v} / \mathrm{v}$, $300 \mathrm{~mL})$, filtered and washed with diethyl ether $(100 \mathrm{~mL})$ to give $\mathbf{1} \cdot 2 \mathrm{HBr}$ as pale yellow crystals; mp $256-258^{\circ} \mathrm{C} ; \alpha_{\mathrm{D}}{ }^{20}=+11.8^{\circ}(\mathrm{c}=1$, MeOH$)$; spectral data were consistent with those published. ${ }^{5}$

1,1-Dimethylethyl (1S,4S)-5-methyl-2,5-diazabicyclo[2.2.1]heptane-2-carboxylate (2). A solution of $1(20.0 \mathrm{~g}, 73 \mathrm{mmol})$ in dry dichloromethane $(200 \mathrm{~mL})$ was cooled to $5{ }^{\circ} \mathrm{C}$ and treated drop-wise with dry triethylamine $(30.0 \mathrm{~g}, 0.29 \mathrm{~mol}, 4$ equiv.). Boc anhydride $(20.0 \mathrm{~g}, 88 \mathrm{mmol}$, 1.25 equiv.) was added, and the mixture was stirred for $6 \mathrm{~h}$. The solvent was removed, the residue was dissolved in water $(100 \mathrm{~mL})$ and extracted continuously with ethyl acetate for $24 \mathrm{~h}$. The organic layer was dried (sodium sulfate) and evaporated. The oily residue was Kugelrohr distilled $\left(60{ }^{\circ} \mathrm{C}, 0.05 \mathrm{mbar}\right)$ to yield a colorless oil 2 (12.0 g, 77\%); $\mathrm{R} f 0.40$ (methanol); ${ }^{1} \mathrm{H}$ NMR $\left(200 \mathrm{MHz}, \mathrm{CDCl}_{3}\right): 1.39(\mathrm{~s}, 9 \mathrm{H}), 1.65(\mathrm{~d}, J=9.5 \mathrm{~Hz}, 1 \mathrm{H}), 1.78(\mathrm{~d}, J=9.5 \mathrm{~Hz}, 1 \mathrm{H}), 2.34(\mathrm{~s}$, $3 \mathrm{H},), 2.47$ (d, $J=9.4 \mathrm{~Hz}, 1 \mathrm{H}), 2.63(\mathrm{~d}, J=9.4 \mathrm{~Hz}, 1 \mathrm{H}), 2.73(\mathrm{~d}, J=9.4 \mathrm{~Hz}, 1 \mathrm{H}), 2.87$ (d, $J=9.4$ $\mathrm{Hz}, 1 \mathrm{H}), 3.10(\mathrm{~d}, J=10.8 \mathrm{~Hz}, 1 \mathrm{H}), 3.26-3.33(\mathrm{~m}, 1 \mathrm{H}), 3.40(\mathrm{~d}, J=10.8 \mathrm{~Hz}, 1 \mathrm{H}), 3.50(\mathrm{~d}, J=$ $10.8 \mathrm{~Hz}, 1 \mathrm{H}), 4.10-4.17(\mathrm{~m}, 1 \mathrm{H}), 4.22-4.31(\mathrm{~m}, 1 \mathrm{H}) ;{ }^{13} \mathrm{C} \mathrm{NMR}\left(50 \mathrm{MHz}, \mathrm{CDCl}_{3}\right)$ : 28.4, 34.9, 36.1, 40.3, 41.2, 48.2, 49.7, 57.2, 58.2, 61.3, 62.3, 62.9, 79.1, 154.2. HRMS Calcd. for $\mathrm{C}_{11} \mathrm{H}_{20} \mathrm{~N}_{2} \mathrm{O}_{2}: 212.1525$. Found: 212.1531 .

\section{Lithiation of 2 followed by reaction with an electrophile. General procedure $A$}

To a solution of TMEDA (778 mg, $7.10 \mathrm{mmol}, 1.5$ equiv.) in dry THF (15 mL) was added $1.3 \mathrm{M}$ sec-BuLi in hexanes $(5.4 \mathrm{~mL}, 7.10 \mathrm{mmol} ; 1.5$ equiv. $)$ at $-78{ }^{\circ} \mathrm{C}$. The mixture was stirred for $30 \mathrm{~min}$. A solution of $2(1.0 \mathrm{~g}, 4.70 \mathrm{mmol})$ in dry THF $(10 \mathrm{~mL})$ was added drop-wise. The solution was stirred for at $-78{ }^{\circ} \mathrm{C}$ for $2 \mathrm{~h}$. The appropriate electrophile (14.1 mmol; 3 equiv.) dissolved in dry THF $(20 \mathrm{~mL})$ was added through a syringe, and the mixture was warmed up to room temperature in the course of $2 \mathrm{~h}$. The reaction was quenched with a saturated aqueous ammonium chloride solution $(40 \mathrm{~mL})$ and stirred for $30 \mathrm{~min}$. The layers were separated, and the aqueous layer was extracted with diethyl ether $(3 \times 50 \mathrm{~mL})$. The organic layers were collected, dried (sodium sulfate) and evaporated to dryness. The residue was purified by flash chromatography, and the product(s) thus obtained were recrystallized.

\section{Lithiation of 2 followed by reaction with an electrophile. General procedure B}

To a solution of TMEDA (778 mg, $7.10 \mathrm{mmol} ; 1.5$ equiv.) in dry diethyl ether (15 mL) was added 1.3 $\mathrm{M}$ sec-BuLi in hexanes $\left(5.4 \mathrm{~mL}, 7.10 \mathrm{mmol} ; 1.5\right.$ equiv.) at $-78{ }^{\circ} \mathrm{C}$, and the mixture was stirred for $30 \mathrm{~min}$. A solution of $2(1.0 \mathrm{~g}, 4.70 \mathrm{mmol})$ in dry diethyl ether $(10 \mathrm{~mL})$ was added drop-wise. After the solution was stirred at $-78{ }^{\circ} \mathrm{C}$ for $2 \mathrm{~h}$, the temperature was allowed to rise 
to $-40{ }^{\circ} \mathrm{C}$ and was kept for $30 \mathrm{~min}$. The mixture was cooled again to $-78{ }^{\circ} \mathrm{C}$, and the appropriate electrophile (14.10 mmol, 3 equiv.) dissolved in dry diethyl ether $(20 \mathrm{~mL})$ was added. The mixture was warmed up to room temperature in the course of $2 \mathrm{~h}$. The reaction was quenched with saturated aqueous ammonium chloride solution $(40 \mathrm{~mL})$ and stirred for $30 \mathrm{~min}$. The layers were separated, and the aqueous layer was extracted with diethyl ether ( 3 x $50 \mathrm{~mL}$ ). The organic layers were combined, dried (sodium sulfate) and evaporated. The residue was purified by flash chromatography and the product(s) thus obtained were recrystallized.

\section{1,1-Dimethylethyl (1S,4S)-1-[hydroxy(diphenyl)methyl]-5-methyl-2,5-diazabi-} cyclo[2.2.1]heptane-2-carboxylate (3a) and 1,1-dimethylethyl (1S,3R,4S)-3-[hydroxy(diphenyl)methyl]-5-methyl-2,5-diazabicyclo[2.2.1]heptane-2-carboxylate (5a). The procedure of lit. ${ }^{9}$ was followed, however, different product mp's were determined: 3a mp 204-206 ${ }^{\circ} \mathrm{C}$ (diisopropyl ether); $5 \mathbf{a} \mathrm{mp}$ (decomp.) $260{ }^{\circ} \mathrm{C}$ (diisopropyl ether).

1,1-Dimethylethyl (1S,4S)-1-[hydroxy[bis[3-(trifluoromethyl)phenyl]]methyl]-5-methyl-2,5diazabicyclo[2.2.1]heptane-2-carboxylate $\quad(3 b), \quad 1,1-d i m e t h y l e t h y l \quad(1 S, 3 S, 4 S)-3-[h y d r o-$ xy[bis[(3-trifluoromethyl)phenyl]]methyl]-5-methyl-2,5-diazabicyclo[2.2.1]heptane-2-

carboxylate (4b) and 1,1-dimethylethyl (1S,3R,4S)-3-[hydroxy[bis[(3-trifluoromethyl)phenyl]]methyl]-5-methyl-2,5-diazabicyclo[2.2.1]heptane-2-carboxylate (5b). Application of Procedure A to bis(3,3'-trifluoromethyl)benzophenone furnished a mixture of $\mathbf{3 b}, \mathbf{4 b}$ and $\mathbf{5 b}$, which was separated by flash chromatography using toluene/diethyl ether (1:1). 3b: Colorless crystals (302 mg, 12\%); mp 190-192 ${ }^{\circ} \mathrm{C}$ (cyclohexane);. Rf 0.25 (toluene/diethyl ether 1:1); []D20 -219.38 (c=0.26, $\left.\mathrm{CHCl}_{3}\right) .{ }^{1} \mathrm{H} \mathrm{NMR}\left(200 \mathrm{MHz}, \mathrm{CDCl}_{3}\right): \delta 1.22(\mathrm{~s}, 9 \mathrm{H}), 1.34(\mathrm{~d}, J=$ $10.2 \mathrm{~Hz}, 1 \mathrm{H}), 2.34$ (s, 3H), 2.38 (d, $J=10.2 \mathrm{~Hz}, 1 \mathrm{H}), 3.08(\mathrm{~d}, J=9.5 \mathrm{~Hz}, 1 \mathrm{H}), 3.11$ (d, $J=9.5$ $\mathrm{Hz}, 1 \mathrm{H}) ; 3.27-3.33$ (m, 1H, H-4), 3.51 (d, $J=12.1 \mathrm{~Hz}, 1 \mathrm{H}, \mathrm{H}-6), 3.90$ (d, J = $12.1 \mathrm{~Hz}, 1 \mathrm{H}, \mathrm{H}-6)$, 7.28-7.92 (m, 8H). ${ }^{13} \mathrm{C}$ NMR (50 MHz, $\left.\mathrm{CDCl}_{3}\right): 26.8,27.8,40.9,42.2,54.0,60.7,61.4,77.2$, 81.1, 122.6, 123.6, 123.9, 124.0, 124.1, 127.8, 128.2, 129.6, 129.8, 130.2, 130.5, 131.1, 144.9, 1456.9, 155.5. Anal. Calcd. for $\mathrm{C}_{26} \mathrm{H}_{28} \mathrm{~F}_{6} \mathrm{~N}_{2} \mathrm{O}_{3}$ : C, 58.87; H, 5.32; N, 5.28. Found: C, 58.90; H, 5.35; N, 5.17. 4b: Colorless crystals (297 mg, 12\%); mp $138-140{ }^{\circ} \mathrm{C}$ (cyclohexane); $\mathrm{R}_{f} 0.13$ (toluene/diethyl ether 1:1). ${ }^{1} \mathrm{H}$ NMR $\left(200 \mathrm{MHz} \mathrm{CDCl}_{3}\right)$ : $1.26(\mathrm{~d}, J=10.2 \mathrm{~Hz}, 1 \mathrm{H}), 1.38-1.57$ $(\mathrm{m}, 10 \mathrm{H}), 2.51(\mathrm{~s}, 3 \mathrm{H}), 2.64-2.76(\mathrm{~m}, 2 \mathrm{H}), 3.32-3.40(\mathrm{~m}, 1 \mathrm{H}), 3.98-4.08(\mathrm{~m}, 1 \mathrm{H}), 4.69-4.80(\mathrm{~m}$, 1H), 7.32-7.82 (m, 8H). ${ }^{13} \mathrm{C} \mathrm{NMR}\left(50 \mathrm{MHz}, \mathrm{CDCl}_{3}\right)$ : 28.0, 31.6, 41.0, 58.8, 61.0, 66.4, 67.7, 78.1, 82.1, 121.2, 123.3, 135.1, 124.2, 124.6, 126.7, 128.0, 128.7, 129.8, 130.5, 131.0, 131.3, 144.5, 147.5, 158.2. Anal. Calcd. for $\mathrm{C}_{26} \mathrm{H}_{28} \mathrm{~F}_{6} 6 \mathrm{~N}_{2} \mathrm{O}_{3}$ : C, 58.87; H, 5.32; N, 5.28. Found: C, 58.94; H, 5.05; N, 5.01. 5b: Colorless oil (104 mg, 4.0\%); Rf 0.70 (toluene/diethyl ether 1:1). ${ }^{1} \mathrm{H}$ NMR (200 MHz, $\left.\mathrm{CDCl}_{3}\right): 1.13(\mathrm{~s}, 9 \mathrm{H}), 1.48(\mathrm{~d}, J=9.5 \mathrm{~Hz}, 1 \mathrm{H}), 1.72(\mathrm{~d}, J=9.5 \mathrm{~Hz}, 1 \mathrm{H}), 2.18$ (s, 3H), 2.38 (dd, $J=11.4,1.9 \mathrm{~Hz}, 1 \mathrm{H}), 3.30-3.37$ (m, 1H), 3.60 (d, $J=11.4 \mathrm{~Hz}, 1 \mathrm{H}), 4.40$ (d, $J$ $=1.8 \mathrm{~Hz}, 1 \mathrm{H}), 4.60-4.70(\mathrm{~m}, 1 \mathrm{H}), 7.20-8.00(\mathrm{~m}, 8 \mathrm{H}) .{ }^{13} \mathrm{C} \mathrm{NMR}\left(50 \mathrm{MHz}, \mathrm{CDCl}_{3}\right): 27.7,33.8$, $42.9,60.0,63.0,66.4,68.0,78.5,80.2,123.2,123.4,123.8,123.9,124.0,127.5,128.6,129.3$, 130.3, 130.4, 130.9, 145.8, 147.6, 157.2. Anal. Calcd. for $\mathrm{C}_{26} \mathrm{H}_{28} \mathrm{~F}_{6} \mathrm{~N}_{2} \mathrm{O}_{3}: \mathrm{C}, 58.87 ; \mathrm{H}, 5.32 ; \mathrm{N}$, 5.28. Found: C, 58.94; H, 5.05; N, 5.01. $\delta$ 


\section{1,1-Dimethylethyl (1S,4S)-1-(9-hydroxy-9H-fluoren-9-yl)-5-methyl-2,5-diaza-} bicyclo[2.2.1]heptane-2-carboxylate (3c) and 1,1-dimethylethyl (1S,3R,4S)-3-(9-hydroxy9H-fluoren-9-yl)-5-methyl-2,5-diazabicyclo[2.2.1]heptane2-carboxylate (5c). Applying Procedure A to fluorenone gave a mixture of $\mathbf{3 c}$ and $\mathbf{5 c}$, which was separated by flash chromatography using toluene/diethyl ether (1:1). 3c: Colorless crystals (480 mg, 26\%); mp 191-193 ${ }^{\circ} \mathrm{C}$ (cyclohexane); $\mathrm{R}_{f} 0.23$ (toluene/diethyl ether 1:1); ${ }^{1} \mathrm{H}$ NMR (200 $\left.\mathrm{MHz}, \mathrm{CDCl}_{3}\right)$ : $0.98(\mathrm{~d}, J=10.2 \mathrm{~Hz}, 1 \mathrm{H}), 1.11(\mathrm{~d}, J=10.2 \mathrm{~Hz}, 1 \mathrm{H}), 1.62(\mathrm{~s}, 9 \mathrm{H}), 2.38(\mathrm{~s}, 3 \mathrm{H}), 2.86-2.94(\mathrm{~m}$, $1 \mathrm{H}), 3.08(\mathrm{~d}, J=8.9 \mathrm{~Hz}, 1 \mathrm{H}), 3.20(\mathrm{~d}, J=10.8 \mathrm{~Hz}, 1 \mathrm{H}), 3.67(\mathrm{~d}, J=8.9 \mathrm{~Hz}, 1 \mathrm{H}), 3.82(\mathrm{~d}, J=$ $10.8 \mathrm{~Hz}, 1 \mathrm{H}), 7.14-7.65(\mathrm{~m}, 8 \mathrm{H}) ;{ }^{13} \mathrm{C} \mathrm{NMR}\left(50 \mathrm{MHz}, \mathrm{CDCl}_{3}\right)$ : 28.3, 39.9, 41.0, 55.1, 59.2, 60.2, $77.4,81.2,82.8,119.3,119.7,124.0,124.9,127.2,127.3,128.3,128.7,139.7,140.0,147.9$, 148.1, 158.0. Anal. Calcd. for $\mathrm{C}_{24} \mathrm{H}_{28} \mathrm{~N}_{2} \mathrm{O}_{3}$ : C, 73.44; H, 7.19; N, 7.14. Found: C, 73.66; H, 7.24; $\mathrm{N}, 7.33$. 5c: Colorless crystals $(250 \mathrm{mg}, 14 \%) ; \mathrm{mp}$ (decomp.) $230{ }^{\circ} \mathrm{C}$ (cyclohexane); $\mathrm{R}_{f} 0.28$ (toluene/diethyl ether 1:1); ${ }^{1} \mathrm{H}$ NMR $\left(200 \mathrm{MHz}, \mathrm{CDCl}_{3}\right): 0.54(\mathrm{~d}, J=9.5 \mathrm{~Hz}, 1 \mathrm{H}), 1.02(\mathrm{~d}, J=$ $9.7 \mathrm{~Hz}, 1 \mathrm{H}), 1.58(\mathrm{~s}, 9 \mathrm{H}), 2.20(\mathrm{~s}, 3 \mathrm{H}), 2.32-2.37(\mathrm{~m}, 1 \mathrm{H}), 2.44(\mathrm{~d}, J=9.4 \mathrm{~Hz}, 1 \mathrm{H}), 2.66(\mathrm{~d}, J=$ $9.4 \mathrm{~Hz}, 1 \mathrm{H}), 4.00-4.06(\mathrm{~m}, 1 \mathrm{H}), 4.48-4.52(\mathrm{~m}, 1 \mathrm{H}), 7.10-7.65(\mathrm{~m}, 8 \mathrm{H}) ;{ }^{13} \mathrm{C} \mathrm{NMR}(50 \mathrm{MHz}$, $\left.\mathrm{CDCl}_{3}\right): \delta 28.3,30.8,33.1,39.8,59.7,60.1,64.9,68.1,81.5,82.5,119.8,119.9,125.2,127.4$, $128.1,128.5,128.8,128.9,139.4,139.7,147.6,147.7,158.3$. Anal. Calcd. for $\mathrm{C}_{24} \mathrm{H}_{28} \mathrm{~N}_{2} \mathrm{O}_{3} .0 .33$ $\mathrm{H}_{2} \mathrm{O}: \mathrm{C}, 72.35 ; \mathrm{H}, 7.25 ; \mathrm{N}, 7.03$. Found: C, 72.45; H, 7.32; N, 7.11.

1,1-Dimethylethyl (1S,4S)-5-Methyl-1-(phenylsulfanyl)-2,5-diazabicyclo-[2.2.1]-heptane-2carboxylate (3d) and 1,1-dimethylethyl (1S,2R,4S)-5-methyl-3-(phenylsulfanyl)-2,5diazabicyclo[2.2.1]heptane-2-carboxylate (5d). Following Procedure B diphenyldisulfide gave a mixture of $\mathbf{3 d}$ and $\mathbf{5 d}$, which was separated by flash chromatography using petroleum ether/ethyl acetate (3:1). 3d: Yellow crystals (503 mg, 33\%); mp 86-88 ${ }^{\circ} \mathrm{C}$ (diisobutyl ether); $\mathrm{R}_{f} 0.40$ (petroleum ether/ethyl acetate 0.40$) .[\alpha]_{\mathrm{D}}{ }^{20}+147.8\left(\mathrm{c}=0.18, \mathrm{CHCl}_{3}\right) ;{ }^{1} \mathrm{H}$ NMR $(200$ $\left.\mathrm{MHz}, \mathrm{CDCl}_{3}\right): 1.50(\mathrm{~s}, 9 \mathrm{H}), 1.68(\mathrm{~d}, J=11.4 \mathrm{~Hz}, 1 \mathrm{H}), 1.81(\mathrm{~d}, J=11.4 \mathrm{~Hz}, 1 \mathrm{H}), 2.36(\mathrm{~s}, 3 \mathrm{H})$, $3.08(\mathrm{~s}, 2 \mathrm{H}), 3.13-3.20(\mathrm{~m}, 1 \mathrm{H}), 3.32(\mathrm{~d}, J=11.0 \mathrm{~Hz}, 1 \mathrm{H}), 3.81(\mathrm{~d}, J=11.0 \mathrm{~Hz}, 1 \mathrm{H}), 7.23-7.38$ $(\mathrm{m}, 3 \mathrm{H}), 7.53-7.61(\mathrm{~m}, 2 \mathrm{H}) ;{ }^{13} \mathrm{C} \mathrm{NMR}\left(50 \mathrm{MHz}, \mathrm{CDCl}_{3}\right)$ : 28.4, 40.7, 44.2, 53.7, 59.7, 65.2, 75.2, 80.5, 128.7, 128.8, 132.3, 136.2, 155.1. HRMS Calcd. for $\mathrm{C}_{17} \mathrm{H}_{24} \mathrm{~N}_{2} \mathrm{O}_{2} \mathrm{~S}: 320.1558$. Found: 320.1567. 5d: Yellow crystals $(510 \mathrm{mg}, 34 \%)$; $\mathrm{mp} 88-90^{\circ} \mathrm{C}\left(\right.$ diisobutyl ether); $\mathrm{R}_{f} 0.15$ (petroleum ether/ethyl acetate $3: 1) ;[\alpha]_{\mathrm{D}}{ }^{20}-109.1\left(\mathrm{c}=0.32, \mathrm{CHCl}_{3}\right) ;{ }^{1} \mathrm{H} \mathrm{NMR}(200 \mathrm{MHz}$, $\left.\mathrm{CDCl}_{3}\right): 1.20(\mathrm{~s}, 9 \mathrm{H}), 2.33(\mathrm{dd}, J=10.7,1.6 \mathrm{~Hz}, 1 \mathrm{H}), 2.46(\mathrm{~s}, 3 \mathrm{H}), 3.24(\mathrm{~d}, J=10.7 \mathrm{~Hz}, 1 \mathrm{H})$, 3.35-3.42 (m, 1H), 4.44-4.55 (m, 1H), 5.03-5.12 (m, 1H), 7.10-7.60 (m, 5H); ${ }^{13} \mathrm{C}$ NMR $(50$ $\left.\mathrm{MHz}, \mathrm{CDCl}_{3}\right)$ : 28.0, 33.1, 44.8, 60.2, 60.9, 69.3, 74.6, 76.4, 77.2, 80.2, 126.6, 127.5, 128.6, 129.0, 131.9, 154.1. HRMS Calcd. for $\mathrm{C}_{17} \mathrm{H}_{24} \mathrm{~N}_{2} \mathrm{O}_{2} \mathrm{~S}: 320.1558$. Found: 320.1569 .

1,1-Dimethylethyl (1S,4S)-1-(9-hydroxy-9H-xanthen-9-yl)-5-methyl-2,5diazabi-cyclo[2.2.1]heptane-2-carboxylate (3e). Following Procedure A and employing 9xanthone the resulting product was isolated and purified by flash chromatography using toluene/diethyl ether (1:1) yielding colorless crystals $3 \mathbf{e}(470 \mathrm{mg}, 25 \%)$; mp 167-168 ${ }^{\circ} \mathrm{C}$ (cyclohexane/petroleum ether $1: 1) ; \mathrm{R}_{f} 0.25$ (toluene/diethyl ether $\left.1: 1\right) ;[\alpha]_{\mathrm{D}}{ }^{20}+63.9(\mathrm{c}=0.21$, $\left.\mathrm{CHCl}_{3}\right)$; ${ }^{1} \mathrm{H}$ NMR $\left(200 \mathrm{MHz}, \mathrm{CDCl}_{3}\right)$ : 1.33 (d, $\left.J=10.2 \mathrm{~Hz}, 1 \mathrm{H}\right), 1.49-1.59$ (m, 10H), 2.25 (s, 
3H), 2.90-3.10 (m, 3H), $3.21(\mathrm{~d}, J=11.4 \mathrm{~Hz}, 1 \mathrm{H}), 3.72(\mathrm{~d}, J=10.2 \mathrm{~Hz}, 1 \mathrm{H}), 7.05-7.35(\mathrm{~m}, 6 \mathrm{H})$, 7.68-7.88 (m, 2H); $\left.{ }^{13} \mathrm{C} \mathrm{NMR} \mathrm{(50} \mathrm{MHz,} \mathrm{CDCl}_{3}\right):$ 28.4, 40.2, 41.0, 55.8, 58.3, 59.2, 70.3, 80.0, 81.5, 115.8, 115.9, 122.8, 123.0, 126.4, 126.6, 126.8, 127.7, 128.4, 128.8, 150.6, 151.7, 158.4. Anal. Calcd. for $\mathrm{C}_{24} \mathrm{H}_{28} \mathrm{~N}_{2} \mathrm{O}_{4}$ : C, 70.57; H, 6.91; N, 6.86. Found: C, 70.50; H, 7.07; N, 6.92.

1,1-Dimethylethyl $(1 S, 3 R, 4 S)-5$-methyl-3-phenylaminocarbonyl-2,5-diazabicyclo[2.2.1]heptane carboxylate (5f). The reaction was conducted following Procedure B except that the lithiated species after stirring for $2 \mathrm{~h}$ was transferred into a solution of phenylisocyanate in dry diethyl ether via a transfer needle. The product was purified by flash chromatography using toluene/diethyl ether/methanol (20:20:1) yielding $\mathbf{5 f}(200 \mathrm{mg}, 13 \%)$ as colorless crystals; mp 191-192 ${ }^{\circ} \mathrm{C}$ (2-propanol); $\mathrm{R}_{f} 0.25$ (toluene/diethyl ether/methanol 20:20:1); ${ }^{1} \mathrm{H}$ NMR (200 MHz, $\left.\mathrm{CDCl}_{3}\right): 1.40(\mathrm{~s}, 9 \mathrm{H}), 1.62(\mathrm{~d}, J=9.5 \mathrm{~Hz}, 1 \mathrm{H}), 1.88(\mathrm{~d}, J=9.5$ $\mathrm{Hz}, 1 \mathrm{H}), 2.32-2.50(\mathrm{~m}, 4 \mathrm{H}), 3.20(\mathrm{~d}, J=10.2 \mathrm{~Hz}, 1 \mathrm{H}), 3.63-3.71(\mathrm{~m}, 1 \mathrm{H}), 4.10-4.15(\mathrm{~m}, 1 \mathrm{H})$, 4.59-4.67 (m, 1H), 7.02-7.56 (m, 5H); $\left.{ }^{13} \mathrm{C} \mathrm{NMR} \mathrm{(50} \mathrm{MHz,} \mathrm{CDCl}_{3}\right)$ : 28.2, 33.6, 44.0, 59.9, 61.7, 66.9, 68.6, 81.1, 119.7, 124.0, 128.9, 137.8, 155.1, 168.6. Anal. Calcd. for $\mathrm{C}_{18} \mathrm{H}_{25} \mathrm{~N}_{3} \mathrm{O}_{3} .0 .11$ $\mathrm{H}_{2} \mathrm{O}: \mathrm{C}, 64.85 ; \mathrm{H}, 7.62 ; \mathrm{N}, 12.60$. Found: C, 64.48; H, 7.25; N, 12.36 .

(5S,7 a $S)-S p i r o-[t e t r a h y d r o-1 H-5,7$ a-methan o-3 H-furo[3,4-a]pyrazin-1,9' [9H] thio-xanthen]-3-one (6). Following Procedure A using 9H-thioxanthen-9-one $(3.0 \mathrm{~g}$, $14.1 \mathrm{mmol})$ the resulting product was purified by flash chromatography using toluene/diethyl ether (1:1) yielding a colorless, viscous oil 6 (403 mg, 24\%); $\mathrm{R}_{f} 0.20$ (toluene:diethyl ether 1:1); ${ }^{1} \mathrm{H}$ NMR (200 MHz, CDCl 3$): 1.22(\mathrm{~d}, J=11.4 \mathrm{~Hz}, 1 \mathrm{H}), 1.47(\mathrm{~d}, J=11.4 \mathrm{~Hz}, 1 \mathrm{H}), 1.98(\mathrm{~d}, J=$ $9.5 \mathrm{~Hz}, 1 \mathrm{H}), 2.25(\mathrm{~s}, 3 \mathrm{H}), 3.04(\mathrm{~d}, J=9.5 \mathrm{~Hz}, 1 \mathrm{H}), 3.32-3.42(\mathrm{~m}, 2 \mathrm{H}), 3.53(\mathrm{~d}, J=8.9 \mathrm{~Hz}, 1 \mathrm{H})$, 7.13-7.82 (m, 8H); $\left.{ }^{13} \mathrm{C} \mathrm{NMR} \mathrm{(50} \mathrm{MHz,} \mathrm{CDCl}_{3}\right)$ : 39.5, 39.9, 47.8, 61.9, 64.6, 80.2, 80.5, 125.2, $125.3,126.7,127.0,128.2,128.3,129.0,129.8,133.4,133.8,157.4$. Anal. Calcd. for $\mathrm{C}_{20} \mathrm{H}_{18} \mathrm{~N}_{2} \mathrm{O}_{2} \mathrm{~S}$ : C, 68.55; H, 5.18; N, 7.99. Found: C, 68.99; H, 5.69; N, 7.44.

(1S,4S)-1-(9-Hydroxy-9H-thioxanthen-9-yl)-2,5-dimethyl-2,5-diazabicyclo

[2.2.1] heptane (7). A solution of $6(300 \mathrm{mg}, 0.86 \mathrm{mmol})$ in dry THF $(10 \mathrm{~mL})$ was cooled to $5{ }^{\circ} \mathrm{C}$ and treated with lithium aluminum hydride $(195 \mathrm{mg}, 5.10 \mathrm{mmol}, 6$ equiv.) in portions. The mixture was stirred at room temperature for $1 \mathrm{~h}$ and hydrolyzed with $2 \mathrm{~N}$ hydrochloric acid. The solution was made alkaline $(\mathrm{pH} 9)$ with $2 \mathrm{~N}$ aqueous potassium hydroxide and extracted with diethyl ether $(3 \times 30 \mathrm{~mL})$. The combined organic layers were dried (sodium sulfate) and evaporated to dryness. The residue was recrystallized from diisopropyl ether to yield 7 (150 mg, $53 \%$ ) as colorless crystals; mp $191-193{ }^{\circ} \mathrm{C}$; $\mathrm{R}_{f} 0.10$ (toluene/diethyl ether:methanol 1:1:1). $[\alpha]_{\mathrm{D}}{ }^{20}+147.7\left(\mathrm{c}=0.17, \mathrm{CHCl}_{3}\right) ;{ }^{1} \mathrm{H} \mathrm{NMR}\left(200 \mathrm{MHz}, \mathrm{CDCl}_{3}\right): 1.13(\mathrm{~d}, J=11.4 \mathrm{~Hz}, 1 \mathrm{H}), 1.78$ $(\mathrm{d}, J=11.4 \mathrm{~Hz}, 1 \mathrm{H}), 2.12,2.18(2 \mathrm{~s}, 6 \mathrm{H}), 2.49(\mathrm{~d}, J=8.9 \mathrm{~Hz}, 1 \mathrm{H}), 3.77-3.82(\mathrm{~m}, 1 \mathrm{H}), 2.93(\mathrm{~d}, J$ $=10.2 \mathrm{~Hz}, 1 \mathrm{H}), 3.04(\mathrm{~d}, J=8.9 \mathrm{~Hz}, 1 \mathrm{H}), 3.26(\mathrm{~d}, J=10.2 \mathrm{~Hz}, 1 \mathrm{H}), 7.14-7.41(\mathrm{~m}, 6 \mathrm{H}), 7.92-$ $8.08(\mathrm{~m}, 2 \mathrm{H}) ;{ }^{13} \mathrm{C} \mathrm{NMR}\left(50 \mathrm{MHz}, \mathrm{CDCl}_{3}\right): \delta$ 39.2, 39.4, 42.3, 55.3, 61.4, 64.1, 72.9, 81.0, 125.9, $126.0,126.1,126.7,127.1,127.1,127.3,128.2,130.9,131.3,137.2,137.6$. Anal. Calcd. for $\mathrm{C}_{20} \mathrm{H}_{22} \mathrm{~N}_{2} \mathrm{O}_{2} \mathrm{~S}: \mathrm{C}, 70.97 ; \mathrm{H}, 6.55 ; \mathrm{N}, 8.28$. Found: C, 70.71; H, 6.59; N, 8.05. 


\section{Boc-Deprotection of 3, 4, 5 with trifluoroacetic acid. General procedure $A$}

A solution of the respective starting material 3, 4, 5 (10\% w/v, 1 equiv.) in dry dichloromethane was cooled to $5{ }^{\circ} \mathrm{C}$ and treated drop-wise with 100 equiv. trifluoroacetic acid. The temperature was allowed to rise to room temperature, and the mixture was stirred for $6 \mathrm{~h}$. The solvent and excess of trifluoroacetic acid was removed, and the residue was suspended in a 100-fold amount of $2 \mathrm{~N}$ aqueous potassium hydroxide. The resulting solid product was filtered off, washed with water until neutral and dried at $50{ }^{\circ} \mathrm{C} / 0.5 \mathrm{mbar}$.

\section{Boc-deprotection of 3, 4, 5 with sodium hydroxide. General procedure B}

A solution of the respective starting material 3, 4, $5(10 \% \mathrm{w} / \mathrm{v}, 1$ equiv.) in ethanol was refluxed with freshly powdered sodium hydroxide (6 equiv.) for $14 \mathrm{~h}$. The solvent was removed and the residue was suspended in water (100-fold amount). The solid product was filtered off, washed with water to neutral $\mathrm{pH}$, and dried at $50{ }^{\circ} \mathrm{C} / 0.5 \mathrm{mbar}$.

(1S,4S)-(5-Methyl-2,5-diazabicyclo[2.2.1]hept-1-yl)diphenylmethanol (8a). Boc-de-protection (Procedure B) of 3a $(500 \mathrm{mg}, 1.27 \mathrm{mmol})$ gave a crude product that was recrystallized from toluene to afford 8a $(170 \mathrm{mg}, 46 \%)$ as colorless crystals; mp $125-126{ }^{\circ} \mathrm{C} ; \mathrm{R}_{f} 0.15$ (chloroform/ethyl acetate/methanol 1:1:0.5); ${ }^{1} \mathrm{H}$ NMR $\left(200 \mathrm{MHz}, \mathrm{CDCl}_{3}\right): 1.85(\mathrm{~d}, J=10.8 \mathrm{~Hz}$, $1 \mathrm{H}), 1.93(\mathrm{~d}, J=10.8 \mathrm{~Hz}, 1 \mathrm{H}), 2.30-2.41(\mathrm{~m}, 4 \mathrm{H}), 2.87$ (d, $J=11.1 \mathrm{~Hz}, 1 \mathrm{H}), 3.19-3.32$ (m, 3H), $3.57(\mathrm{~d}, J=11.1 \mathrm{~Hz}, 1 \mathrm{H}), 7.18-7.68(\mathrm{~m}, 10 \mathrm{H}) ;{ }^{13} \mathrm{C} \mathrm{NMR}\left(50 \mathrm{MHz}, \mathrm{CDCl}_{3}\right): 37.8,40.0,62.3$, 65.3, 75.5, 76.9, 126.8, 126.9, 127.0, 127.3, 127.7, 127.9, 143.8, 145.6. Anal. Calcd. for $\mathrm{C}_{19} \mathrm{H}_{22} \mathrm{~N}_{2} \mathrm{O}$ : C, 77.52; H, 7.53; N, 9.52. Found: C, 77.52; H, 7.65; N, 9.51.

\section{$(1 S, 4 S)-(5-M e t h y l-2,5-d i a z a b i c y c l o[2.2 .1]$ hept-1-yl)[bis[3-(trifluoromethyl)phenyl]]-}

methanol (8b). Boc-Deprotection (Procedure A) of 3b (100 mg, $0.23 \mathrm{mmol}$ ) gave an oily crude product that was extracted with diethyl ether $(3 \times 10 \mathrm{~mL})$. The organic layers were combined, dried (sodium sulfate) and evaporated to dryness to yield $\mathbf{8 b}\left(65 \mathrm{mg}, 81 \%\right.$ ) as a colorless oil; $\mathrm{R}_{f}$ 0.20 (toluene/diethyl ether/methanol 1:1:1); ${ }^{1} \mathrm{H} \mathrm{NMR}\left(200 \mathrm{MHz}, \mathrm{CDCl}_{3}\right): 1.20(\mathrm{~d}, J=9.5 \mathrm{~Hz}$, $1 \mathrm{H}), 1.88(\mathrm{~d}, J=9.5 \mathrm{~Hz}, 1 \mathrm{H}), 2.38(\mathrm{~s}, 3 \mathrm{H}), 2.54(\mathrm{~d}, J=11.4 \mathrm{~Hz}, 1 \mathrm{H}), 2.91(\mathrm{~d}, J=11.1 \mathrm{~Hz}, 1 \mathrm{H})$, 3.28-3.37 (m, 2H), $3.50(\mathrm{~d}, J=11.1 \mathrm{~Hz}, 1 \mathrm{H}), 7.33-8.00(\mathrm{~m}, 8 \mathrm{H}) ;{ }^{13} \mathrm{C}$ NMR $\left(50 \mathrm{MHz}, \mathrm{CDCl}_{3}\right)$ : 15.2, 29.7, 37.6, 39.7, 47.0, 62.4, 64.2, 65.8, 75.3, 76.6, 121.3, 123.8, 123.9, 124.0, 124.3, 124.4, 126.7, 128.4, 128.6, 130.1, 130.3, 130.5, 130.8, 131.0, 144.2, 145.4). HRMS Calcd. for $\mathrm{C}_{21} \mathrm{H}_{20} \mathrm{~N}_{2} \mathrm{OF}_{6}$ : 430.1480. Found: 430.1495 .

(1S,4S)-1-(9-Hydroxy-9H-fluoren-9-yl)-5-methyl-2,5-diazabicyclo[2.2.1]heptane (8c). Bocdeprotection (Procedure A) of 3c (330 mg, $0.84 \mathrm{mmol})$ gave 8c $(198 \mathrm{mg}, 82 \%)$ as colorless crystals; mp $258-261{ }^{\circ} \mathrm{C}$; $\mathrm{R}_{f} 0.03$ (toluene:diethyl ether, $\left.1: 1\right) ;[\alpha]_{\mathrm{D}}{ }^{20}+24.5\left(\mathrm{c}=0.20, \mathrm{CHCl}_{3}\right) ;{ }^{1} \mathrm{H}$ NMR (200 MHz, CDCl $): 1.73$ (d, $J=9.8 \mathrm{~Hz}, 1 \mathrm{H}, \mathrm{H}-7), 1.97$ (d, $J=10.2 \mathrm{~Hz}, 1 \mathrm{H}, \mathrm{H}-6), 2.08-$ 2.18 (m, 4H, N-CH 3 , H-7), 2.28 (d, $J=10.2 \mathrm{~Hz}, 1 \mathrm{H}, \mathrm{H}-6), 3.00$ (d, J=10.8 Hz, 1H, H-3), 3.23$3.30(\mathrm{~m}, 1 \mathrm{H}, \mathrm{H}-1), 3.26$ (d, $J=10.8 \mathrm{~Hz}, 1 \mathrm{H}, \mathrm{H}-3), 7.22-7.66\left(\mathrm{~m}, 8 \mathrm{H}, \mathrm{H}_{\text {arom }}\right) ;{ }^{13} \mathrm{C} \mathrm{NMR}(50 \mathrm{MHz}$, $\left.\mathrm{CDCl}_{3}\right): 29.7,36.6,48.3,63.6,63.7,73.5,77.0,81.1,119.8,120.2,124.0,125.0,127.4,128.8$, 129.2, 139.4, 140.5, 146.0, 148.3. Anal. Calcd. for $\mathrm{C}_{19} \mathrm{H}_{20} \mathrm{~N}_{2} \mathrm{O} .0 .33 \mathrm{H} 2 \mathrm{O}: \mathrm{C}, 76.45 ; \mathrm{H}, 6.98 ; \mathrm{N}$, 9.38. Found: C, 76.53; H, 6.97; N, 9.26. 


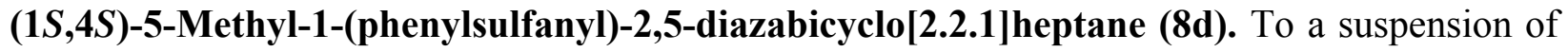
lithium aluminum hydride (142 mg, $3.7 \mathrm{mmol}, 8$ equiv.) in dry THF (5 mL) 3d (150 mg, $0.47 \mathrm{mmol}$ ) was added in portions. The mixture was stirred at $50{ }^{\circ} \mathrm{C}$ for $12 \mathrm{~h}$, hydrolyzed with 2 $\mathrm{N}$ hydrochloric acid and extracted with diethyl ether $(20 \mathrm{~mL})$. The aqueous layer was made alkaline ( $\mathrm{pH} 8$ ) with conc. ammonia and extracted with diethyl ether ( $3 \times 20 \mathrm{~mL})$. The combined organic layers were dried (sodium sulfate) and evaporated to dryness, yielding 8d (20 mg, 18\%) as colorless oil; $\mathrm{R}_{f} 0.50$ (petroleum ether/ethyl acetate/methanol 1:1:1); ${ }^{1} \mathrm{H}$ NMR (200 MHz, $\left.\mathrm{CDCl}_{3}\right): 1.82(\mathrm{~d}, J=7.6 \mathrm{~Hz}, 1 \mathrm{H}), 1.90(\mathrm{~d}, J=7.6 \mathrm{~Hz}, 1 \mathrm{H}), 2.50$ (s, 3H), 2.54-2.68 (m, 2H), 3.00-3.18 (m, 3H), 7.23-7.40 (m, 3H), 7.5-7.63 (m, 2H); $\left.{ }^{13} \mathrm{C} \mathrm{NMR} \mathrm{(50} \mathrm{MHz,} \mathrm{CDCl}_{3}\right)$ : 35.0, 41.2, 41.7, 58.0, 58.2, 61.6, 78.9, 128.0, 128.5, 134.8. HRMS Calcd. for $\mathrm{C}_{12} \mathrm{H}_{16} \mathrm{~N}_{2} \mathrm{~S}: 220.1034$. Found: 220.1026.

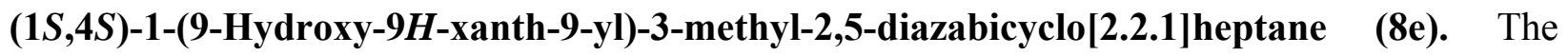
Boc-deprotection (Procedure A) of 3e (340 mg, $1.10 \mathrm{mmol})$ yielded $\mathbf{8 e}(50 \mathrm{mg}, 20 \%)$ as colorless crystals; mp 178-179 ${ }^{\circ} \mathrm{C}$; Rf 0.10 (toluene/diethyl ether/methanol 1:1:1); ${ }^{1} \mathrm{H}$ NMR (200 MHz, $\left.\mathrm{CDCl}_{3}\right): 1.34(\mathrm{~d}, J=9.5 \mathrm{~Hz}, 1 \mathrm{H}), 1.63(\mathrm{~d}, J=9.8 \mathrm{~Hz}, 1 \mathrm{H}), 1.76(\mathrm{~d}, J=9.5 \mathrm{~Hz}, 1 \mathrm{H}), 2.12(\mathrm{~s}$, $3 \mathrm{H}), 2.70(\mathrm{~d}, J=9.8 \mathrm{~Hz}, 1 \mathrm{H}), 2.85(\mathrm{~d}, J=11.4 \mathrm{~Hz}, 1 \mathrm{H}), 3.00-3.10(\mathrm{~m}, 1 \mathrm{H}), 3.18(\mathrm{~d}, J=11.4$ $\mathrm{Hz}, 1 \mathrm{H}), 7.03-7.78(\mathrm{~m}, 8 \mathrm{H}) ;{ }^{13} \mathrm{C} \mathrm{NMR}\left(50 \mathrm{MHz}, \mathrm{CDCl}_{3}\right)$ : 35.9, 40.4, 48.2, 62.9, 63.4, 67.6, 76.6, 116.0, 116.2, 123.0, 123.1, 124.7, 126.1, 126.2, 127.1, 128.5, 128.7, 150.5, 151.3. HRMS Calcd. for $\mathrm{C}_{19} \mathrm{H}_{20} \mathrm{~N}_{2} \mathrm{O}_{2}$ : 308.1525. Found: 308.1518 .

\section{(1S,3S,4S)-(5-Methyl-2,5-diazabicyclo[2.2.1]hept-3-yl)[bis[3-(trifluoromethyl)phenyl]]-}

methanol (9b). Following Procedure B 4b (70 mg, $0.13 \mathrm{mmol})$ gave 9b (40 mg, 71\%) as colorless crystals; mp $62-64{ }^{\circ} \mathrm{C} ; \mathrm{R}_{f} 0.25$ (toluene/diethyl ether/methanol, 1:1:1); ${ }^{1} \mathrm{H}$ NMR (200 $\mathrm{MHz}_{\mathrm{CDCl}}$ ): 1.45 (d, $\left.J=11.1 \mathrm{~Hz}, 1 \mathrm{H}, \mathrm{H}-7\right), 1.87$ (d, J=11.1 Hz, 1H, H-7), 2.32 (s, 9H, N$\mathrm{CH}_{3}$ ), 2.59 (d, $\left.J=9.5 \mathrm{~Hz}, 1 \mathrm{H}, \mathrm{H}-6\right), 2.70$ (d, $\left.J=9.5 \mathrm{~Hz}, 1 \mathrm{H}, \mathrm{H}-6\right), 2.78-2.83$ (m, 1H, H-1), 3.58-3.64 (m, 1H, H-4), 4.15-4.25 (m, 1H, H-3), 7.32-7.88 (m, 8H, arom. H's); ${ }^{13} \mathrm{C}$ NMR (50 $\left.\mathrm{MHz}, \mathrm{CDCl}_{3}\right)$ : 31.7, 41.3, 56.9, 63.4, 64.3, 64.7, 76.2, 121.4, 122.2, 122.3, 122.7, 123.8, 126.8, 128.8, 129.0, 129.5, 130.4, 131.0, 145.1, 148.4; HRMS Calcd. for $\mathrm{C}_{21} \mathrm{H}_{20} \mathrm{~N}_{2} \mathrm{OF}_{6}$ : 430.1480 . Found: 430.1480 .

(1S,3S,4S)-3-(9-Hydroxy-9H-fluoren-9-yl)-5-methyl-2,5-diazabicyclo[2.2.1]heptane (9c). Boc-deprotection (Procedure A) of 3c $(330 \mathrm{mg}, 0.84 \mathrm{mmol})$ gave $9 \mathbf{c}(198 \mathrm{mg}, 82 \%)$ as colorless crystals; mp $258-261{ }^{\circ} \mathrm{C}$; Rf 0.03 (toluene/diethyl ether $\left.1: 1\right) ;[\alpha]_{\mathrm{D}}{ }^{20}+24.5\left(\mathrm{c}=0.20, \mathrm{CHCl}_{3}\right) ;{ }^{1} \mathrm{H}$ NMR (200 MHz, $\left.\mathrm{CDCl}_{3}\right): 1.73(\mathrm{~d}, J=9.8 \mathrm{~Hz}, 1 \mathrm{H}), 1.97(\mathrm{~d}, J=10.2 \mathrm{~Hz}, 1 \mathrm{H}), 2.08-2.18(\mathrm{~m}$, 4H), $2.28(\mathrm{~d}, J=10.2 \mathrm{~Hz}, 1 \mathrm{H}), 3.00(\mathrm{~d}, J=10.8 \mathrm{~Hz}, 1 \mathrm{H}), 3.23-3.30(\mathrm{~m}, 1 \mathrm{H}), 3.26(\mathrm{~d}, J=10.8$ $\mathrm{Hz}, 1 \mathrm{H}), 7.22-7.66(\mathrm{~m}, 8 \mathrm{H}) ;{ }^{13} \mathrm{C} \mathrm{NMR}\left(50 \mathrm{MHz}, \mathrm{CDCl}_{3}\right)$ : 29.7, 36.6, 48.3, 63.6, 63.7, 73.5, 77.0, 81.1, 119.8, 120.2, 124.0, 125.0, 127.4, 128.8, 129.2, 139.4, 140.5, 146.0, 148.3. Anal. Calcd. for $\mathrm{C}_{19} \mathrm{H}_{20} \mathrm{~N}_{2} \mathrm{O} .0 .33 \mathrm{H}_{2} \mathrm{O}: \mathrm{C}, 76.45 ; \mathrm{H}, 6.98 ; \mathrm{N}, 9.38$. Found: C, 76.53; H, 6.97; N, 9.26.

(1S,3R,4S)-(5-Methyl-2,5-diazabicyclo[2.2.1]hept-3-yl)diphenylmethanol (10a). Boc-deprotection (Procedure B) of $\mathbf{5 a}(200 \mathrm{mg}, 0.51 \mathrm{mmol})$ gave a crude product that was recrystallized from toluene yielding $\mathbf{1 0 a}(120 \mathrm{mg}, 80 \%)$ as colorless crystals; mp $135-136{ }^{\circ} \mathrm{C}$. $\mathrm{R}_{f} 0.10$ (chloroform/ethyl acetate/methanol 1:1:0.5); ${ }^{1} \mathrm{H}$ NMR (200 MHz, $\left.\mathrm{CDCl}_{3}\right): 1.48$ (d, $J=10.2 \mathrm{~Hz}$, 
1H), 1.90 (d, $J=10.2 \mathrm{~Hz}, 1 \mathrm{H}), 2.33(\mathrm{~s}, 3 \mathrm{H}), 2.53$ (d, $J=9.8 \mathrm{~Hz}, 1 \mathrm{H}), 2.74(\mathrm{~d}, J=9.8 \mathrm{~Hz}, 1 \mathrm{H})$, $1.85-1.91(\mathrm{~m}, 1 \mathrm{H}), 3.53-3.60(\mathrm{~m}, 1 \mathrm{H}), 4.20-4.28(\mathrm{~m}, 1 \mathrm{H}), 7.10-7.58(\mathrm{~m}, 10 \mathrm{H}) ;{ }^{13} \mathrm{C}$ NMR $(50$ $\mathrm{MHz}_{\mathrm{CDCl}}$ ): 29.7, 32.2, 41.0, 56.8, 63.5, 65.0, 76.7, 125.5, 126.0, 126.5, 127.9, 128.2, 144.7, 147.8. Anal. Calcd. for $\mathrm{C}_{19} \mathrm{H}_{22} \mathrm{~N}_{2} \mathrm{O}$ : C, 77.52; H, 7.53; N, 9.52. Found: C, 77.76; H, 7.72; N, 9.55 .

(1S,3R,4S)-(5-Methyl-2,5-diazabicyclo[2.2.1]heptyl)[bis[3-(trifluoromethyl)phenyl]]methanol (10b). Boc-deprotection (Procedure A) of $\mathbf{5 b}(70 \mathrm{mg}, 0.13 \mathrm{mmol})$ gave $\mathbf{1 0 b}(40 \mathrm{mg}$, $71 \%$ ) as colorless crystals; mp $62-64{ }^{\circ} \mathrm{C}$; $\mathrm{R}_{f} 0.25$ (toluene/diethyl ether/methanol 1:1:1); ${ }^{1} \mathrm{H}$ NMR (200 MHz, $\left.\mathrm{CDCl}_{3}\right): 1.45(\mathrm{~d}, J=11.1 \mathrm{~Hz}, 1 \mathrm{H}), 1.87(\mathrm{~d}, J=11.1 \mathrm{~Hz}, 1 \mathrm{H}), 2.32(\mathrm{~s}, 9 \mathrm{H})$, $2.59(\mathrm{~d}, J=9.5 \mathrm{~Hz}, 1 \mathrm{H}), 2.70(\mathrm{~d}, J=9.5 \mathrm{~Hz}, 1 \mathrm{H}), 2.78-2.83(\mathrm{~m}, 1 \mathrm{H}), 3.58-3.64(\mathrm{~m}, 1 \mathrm{H}), 4.15-$ $4.25(\mathrm{~m}, 1 \mathrm{H}), 7.32-7.88(\mathrm{~m}, 8 \mathrm{H}) ;{ }^{13} \mathrm{C} \mathrm{NMR}\left(50 \mathrm{MHz}, \mathrm{CDCl}_{3}\right)$ : 31.7, 41.3, 56.9, 63.4, 64.3, 64.7, 76.2, 121.4, 122.2, 122.3, 122.7, 123.8, 126.8, 128.8, 129.0, 129.5, 130.4, 131.0, 145.1, 148.4. HRMS: Calcd. for $\mathrm{C}_{21} \mathrm{H}_{20} \mathrm{~N}_{2} \mathrm{OF}_{6}$ : 430.1480. Found: 430.1480 .

\section{Asymmetric reduction of propiophenone. General procedure}

A solution (in some cases a suspension) of the ligands $\mathbf{8 a}, \mathbf{8 b}$, or $\mathbf{8 c}(0.10 \mathrm{mmol})$ in dry THF $(1 \mathrm{~mL})$ was treated with trimethyl borate $(14 \mathrm{~L}, 0.12 \mathrm{mmol})$ and was stirred for $1 \mathrm{~h}$. To this mixture was added borane dimethylsulfide (neat; $0.1 \mathrm{~mL}, 1.00 \mathrm{mmol}$ ), and the mixture was stirred for further $10 \mathrm{~min}$. With a syringe propiophenone $(133 \mathrm{~L}, 1.00 \mathrm{mmol})$ was added over a period of $30 \mathrm{~min}$, and the mixture was stirred until completion (as monitored by TLC)of the reaction (usually $30 \mathrm{~min}$ after addition of propiophenone). After $2 \mathrm{~N}$ hydrochloric acid (5 $\mathrm{mL}$ ) was added, the layers were separated, and the aqueous layer was extracted with diethyl ether ( $3 \mathrm{x}$ $5 \mathrm{~mL}$ ). The organic combined organic layers were dried (sodium sulfate) and evaporated to dryness. The resulting colorless oil was separated by chiral HPLC. $\operatorname{tr}(R)-1$-phenylpropanol: 14 min; $\operatorname{tr}(S)$-1-phenylpropanol: 16 min; cf. Table 3.

\section{Acknowledgements}

We want to thank Dipl.-Ing. M. Treu and Dipl.-Ing. C. Laggner for synthetic contributions and inspiring discussions. We were grateful to Sanochemia for a donation of chemicals.

\section{References}

1. (a) Portoghese, P. S.; Mikhail, A. A. J. Org. Chem. 1966, 31, 1059. (b) Sepulchre, A.-M.; Cleophax, J.; Hildesheim, J.; Gero, S. D. C. R. Acad. Sc. Paris C 1969, 268, 849. (c) Portoghese, P. S.; Sepp, D. T. J. Heterocycl. Chem. 1971, 8, 531. (d) Sturm, P. A.; Henry, D. W. J. Med. Chem. 1974, 17, 481. (e) Rosen, T.; Chu, D. T. W. Lico, I. M.; Fernandes, P. 
B.; Marsh, K.; Shen, L.; Cepa, V. G.; Pernet, A. G. J. Med. Chem. 1988, 31, 1598. (f) Rosen, T.; Lico, I. M.; Chu, D. T. W. J. Org. Chem. 1988, 53, 1580. (g) Jordis, U.; Sauter, F.; Siddiqi, S.; Küenburg, B.; Bhattacharya, K. Synthesis 1990, 10, 925. (h) Bouzard, D.; Di Cesare, P.; Esiz, M.; Jacquet, J. P.; Kiechel, J. R.; Remuzon, P.; Weber, A.; Oki, T.; Masuyoshi, M.; Kessler, R. E.; Fung-Tomc, J.; Desiderio, J. J. Med. Chem. 1990, 33, 1344. (i) Kiely, J. S.; Hutt, M. P.; Culbertson, T. P.; Bucsh, R. A.; Worth, D. F.; Lesheski, L, E.; Gogliotti, R. D.; Sesnie, J. C.; Solomon, M.; Mich, T. F. J. Med. Chem. 1991, 34, 656. (j) Remuzon, P.; Bouzard, D.; Guiol, C.; Jacquet, J. J. Med. Chem. 1992, 35, 2898. (k) Polonski, T. J. Org. Chem. 1993, 58, 258. (1) Li, Qun; Chu, D. T. W.; Claiborne; A.; Cooper, C. S.; Lee, C. M.; Raye, K.; Berst, K. B.; Donner, P.; Wang, W.; Hasvold, L.; Fung, A.; Zhenkun, M.; Tufano, R. F.; Linus, L. S.; Baranowski, J.; Nilius, A.; Alder, J.; Meulbroek, J.; Marsh, K.; Crowell, D.; Hui, Y.; Seif, L.; Melcher, L. M.; Henry, R.; Spanton, S.; Faghih, R.; Klein, L. L.; Tanaka, K., S.; Plattner, J. J. J. Med. Chem. 1996, 39, 3070. (m) Rajeev, K. G.; Sanjayan, G. J.; Ganesh, K. N. J. Org. Chem. 1997, 62, 5169.

2. (a) Remuzon, P.; Massoudi, M.; Bouzard, D.; Jacquet, J.-P. Heterocycles 1992, 34, 679. (b) Remuzon, P.; Bouzard, D.; Clemencin, C.; Dussy, C.; Essiz, M.; Jacquet, J.-P.; SaintGermain, J. J. Heterocyclic Chem. 1993, 30, 517. (c) Remuzon, P.; Bouzard, D.; Dussy, C.; Jacquet, J.-P.; Massoudi, M. Heterocycles 1992, 34, 241.

3. (a) Abraham, D. J.; Mokotoff, M.; Sheh, L.; Simmons, J. E. J. Med. Chem. 1983, 26, 549.

(b) Hadfield, P. S.; Galt, R. H. B.; Sawyer, Y.; Layland, N. J.; Page, M. I. J. Chem. Soc., Perkin Trans. 1 1997, 503. (c) Andreatta, R. H.; Nair, V.; Robertson, A. V.; Simpson, W. R. J. Aust. J. Chem. 1967, 20, 1493.

4. Hultin, P. G.; Szarek, W. A. Can. J. Chem. 1994, 72, 1978.

5. (a) Braish, T. F.; Darrell, E. F. J. Org. Chem. 1990, 55, 1684. (b) Braish, T. F.; Darrell, E. F. U.S. Patent 5.196.548, 1992; Chem. Abstr. 1991, 114, 247308.

6. Beak, P.; Zajdel, W. J.; Reitz, D. B. Chem. Rev. 1984, 84, 471.

7. Hoppe, D.; Hintze, F.; Tebben, P.; Paetow, M.; Aherns, H.; Schwerdtfeger, J.; Sommerfeld, P.; Haller, J.; Guarnieri, W.; Kolczewski, S.; Hense, T.; Hoppe, I. Pure Appl. Chem. 1994, 50, 1479.

8. Tsukazaki, M.; Tinkl, M.; Roglans, A.; Chapell, B. J.; Taylor, N. J.; Snieckus, V. J. Am. Chem. Soc. 1996, 118, 685.

9. Jordis, U.; Kesselgruber, M.; Nerdinger, S.; Mereiter, K. Mendeleev Commun. 1999, 147.

10. For a review on the asymmetric addition of dialkylzinc reagents to aldehydes see: Soai, K.; Niwa S. Chem. Rev. 1992, 92, 833.

11. Corey, E. J.; Bakshi, R. K.; Shibata, S. J. Am. Chem. Soc. 1987, 109, 5551.

12. For an example see: Agami, C.; Amiot, F.; Couty, F.; Dechoux, L.; Kaminsky, C.; Venier, O. Tetrahedron Asymmetry 1998, 9, 3955. 